\title{
Numerical Solution for the Fractional Wave Equation Using Pseudo-Spectral Method Based on the Generalized Laguerre Polynomials
}

\author{
Nasser H. Sweilam1, Mohamed M. Khader',3, Mohamed Adel1 \\ ${ }^{1}$ Department of Mathematics, Faculty of Science, Cairo University, Giza, Egypt \\ ${ }^{2}$ Department of Mathematics and Statistics, College of Science, Al-Imam Mohammad Ibn Saud Islamic \\ University (IMSIU), Riyadh, Kingdom of Saudi Arabia \\ ${ }^{3}$ Department of Mathematics, Faculty of Science, Benha University, Benha, Egypt \\ Email: nsweilam@sci.cu.edu.eg, mohamedmbd@yahoo.com
}

Received 15 February 2015; accepted 3 April 2015; published 10 April 2015

Copyright (C) 2015 by authors and Scientific Research Publishing Inc.

This work is licensed under the Creative Commons Attribution International License (CC BY). http://creativecommons.org/licenses/by/4.0/

(c)

\begin{abstract}
In this paper, an efficient numerical method is considered for solving the fractional wave equation (FWE). The fractional derivative is described in the Caputo sense. The method is based on Laguerre approximations. The properties of Laguerre polynomials are utilized to reduce FWE to a system of ordinary differential equations, which is solved by the finite difference method. An approximate formula of the fractional derivative is given. Special attention is given to study the convergence analysis and estimate an error upper bound of the presented formula. Numerical solutions of FWE are given and the results are compared with the exact solution.
\end{abstract}

\section{Keywords}

Fractional Wave Equation, Caputo Derivative, Finite Difference Method, Laguerre Polynomials, Convergence Analysis

\section{Introduction}

The subject of fractional calculus was planted over 300 year ago. The theory of derivative and integrals of noninteger order goes back to Liouville, Leibnitz, Grunwald-Letnikov, Reimann and Letnikov. In the recent years, fractional calculus has played a very significant role in many areas in fluid flow, mechanics, viscoelasticity, bi- 
ology, physics, science and engineering, and other applications [1]. Fractional derivatives provide an excellent instrument for the description of memory and hereditary properties of various materials and processes. Half-order derivatives and integrals are proved to be more useful for the formulation of certain electrochemical problems than the classical models [2]. Thus, seeking solutions of nonlinear fractional differential equations (FDEs) is still a significant task. Except in a limited numbers of these equations, we have difficulty to find their analytical as well as approximate solutions. Therefore, there have been attempts to develop the new methods for obtaining analytical and approximate solutions of nonlinear FDEs. Recently, several methods have drawn special attention, such as homotopy perturbation method [3], homotopy analysis method [4], collocation method ([5]-[9]) and finite difference method ([10] [11]).

Our main goal in this paper is concerned with the application of Laguerre pseudo-spectral method to obtain the numerical solution of FWE of the following form

$$
u_{t t}(x, t)=d(x, t) D^{v} u(x, t)+s(x, t), \quad 0<x<a, \quad 0 \leq t \leq T,
$$

here the parameter $v$ refers to the fractional order of spatial derivatives with $1<v \leq 2$. The function $s(x, t)$ is a source term and $d(x, t)$ is the coefficient function which is a given continues function satisfies Lipschitz condition. We also assume the following initial conditions

$$
u(x, 0)=u^{0}(x), \quad u_{t}(x, 0)=u^{1}(x), \quad 0<x<a,
$$

and the following Dirichlet boundary conditions

$$
u(0, t)=u(a, t)=0 .
$$

Note that at $v=2$, Equation (1) is the classical wave equation

$$
u_{t t}(x, t)=d(x, t) u_{x x}(x, t)+s(x, t) .
$$

Many authors studied the numerical solutions of the introduced problem (1) using different numerical methods such as, Adomian decomposition method [12] and finite difference methods ([13] [14]) and others.

Our idea is to apply the Laguerre collocation method to discretize (1) to get a linear system of ordinary differential equations (ODEs) thus greatly simplifying the problem, and use the finite difference method (FDM) ([15]-[18]) to solve the resulting system.

The structure of this paper is arranged in the following way: In Section 2, we introduce some basic definitions about Caputo fractional derivatives and properties of the generalized Laguerre polynomials. In Section 3, we introduce the fundamental theorems for the fractional derivatives of the generalized Laguerre polynomials and its convergence analysis. In Section 4, we give the procedure of solution for FWE. In Section 5, numerical example is given to solve FWE and show the accuracy of the presented method. Finally, in Section 6, the paper ends with a brief conclusion and some remarks.

\section{Preliminaries and Notations}

In this section, we present some necessary definitions and mathematical preliminaries of the fractional calculus theory required for our subsequent development.

\subsection{The Caputo Fractional Derivative}

Definition1.

The Caputo fractional derivative operator $D^{\alpha}$ of order $\alpha$ is defined in the following form

$$
D^{v} f(x)=\frac{1}{\Gamma(m-v)} \int_{0}^{x} \frac{f^{(m)}(t)}{(x-t)^{v-m+1}} \mathrm{~d} t, \quad v>0, \quad x>0,
$$

where $m-1<v \leq m, m \in \mathbb{N}, \Gamma($.$) is the gamma function.$

Similar to integer-order differentiation, Caputo fractional derivative operator is a linear operation

$$
D^{v}(\lambda f(x)+\mu g(x))=\lambda D^{v} f(x)+\mu D^{v} g(x),
$$


where $\lambda$ and $\mu$ are constants. For the Caputo's derivative we have $D^{v} C=0, C$ is a constant and

$$
D^{v} x^{n}= \begin{cases}0, & \text { for } n \in \mathbb{N}_{0} \text { and } n<\lceil v\rceil ; \\ \frac{\Gamma(n+1)}{\Gamma(n+1-v)} x^{n-v}, & \text { for } n \in \mathbb{N}_{0} \text { and } n \geq\lceil v\rceil .\end{cases}
$$

We use the ceiling function $\lceil v\rceil$ to denote the smallest integer greater than or equal to $v$, and $\mathbb{N}_{0}=\{0,1,2, \cdots\}$. Recall that for $v \in \mathbb{N}$, the Caputo differential operator coincides with the usual differential operator of integer order. For more details on fractional derivatives definitions and its properties see ([2] [19]).

\subsection{The Definition and Properties of the Generalized Laguerre Polynomials}

The generalized Laguerre polynomials $\left[L_{n}^{(\alpha)}(x)\right]_{n=0}^{\infty}, \alpha>-1$ are defined on the unbounded interval $(0, \infty)$ and can be determined with the aid of the following recurrence formula

$$
(n+1) L_{n+1}^{(\alpha)}(x)+(x-2 n-\alpha-1) L_{n}^{(\alpha)}(x)+(n+\alpha) L_{n-1}^{(\alpha)}(x)=0, \quad n=1,2, \cdots,
$$

where, $L_{0}^{(\alpha)}(x)=1$ and $L_{1}^{(\alpha)}(x)=\alpha+1-x$.

The analytic form of these polynomials of degree $n$ is given by [20]

$$
L_{n}^{(\alpha)}(x)=\left(\begin{array}{c}
n+\alpha \\
n
\end{array}\right) \sum_{k=0}^{n} \frac{(-n)_{k}}{(\alpha+1)_{k}}=\frac{x^{k}}{k !} \sum_{k=0}^{n} \frac{(-1)^{k}}{k !}\left(\begin{array}{c}
n+\alpha \\
n-k
\end{array}\right) x^{k},
$$

$L_{n}^{(\alpha)}(0)=\left(\begin{array}{c}n+\alpha \\ n\end{array}\right)$. These polynomials are orthogonal on the interval $[0, \infty)$ with respect to the weight function $w(x)=\frac{1}{\Gamma(1+\alpha)} x^{\alpha} \mathrm{e}^{-x}$. The orthogonality relation is

$$
\frac{1}{\Gamma(1+\alpha)} \int_{0}^{\infty} x^{\alpha} \mathrm{e}^{-x} L_{m}^{(\alpha)}(x) L_{n}^{(\alpha)}(x) \mathrm{d} x=\left(\begin{array}{c}
n+\alpha \\
n
\end{array}\right) \delta_{m n} .
$$

Also, they satisfy the differentiation formula

$$
D^{k} L_{n}^{(\alpha)}(x)=(-1)^{k} L_{n-k}^{(\alpha+k)}(x), \quad k=0,1, \cdots, n .
$$

Any function $u(x)$ belongs to the space $L_{w}^{2}[0, \infty)$ of all square integrable functions on $[0, \infty)$ with weight function $w(x)$, can be expanded in the following Laguerre series

$$
u(x)=\sum_{n=0}^{\infty} c_{n} L_{n}^{(\alpha)}(x),
$$

where the coefficients $c_{n}$ are given by

$$
c_{n}=\frac{\Gamma(n+1)}{\Gamma(n+\alpha+1)} \int_{0}^{\infty} x^{\alpha} \mathrm{e}^{-x} L_{n}^{(\alpha)}(x) u(x) \mathrm{d} x, \quad n=0,1, \cdots .
$$

Consider only the first $(m+1)$ terms of generalized Laguerre polynomials, so we can write

$$
u_{m}(x)=\sum_{n=0}^{m} c_{n} L_{n}^{(\alpha)}(x) .
$$

For more details on Laguerre polynomials, its definitions and properties see ([21] [22]).

\section{The Approximate Fractional Derivatives of $L_{n}^{(\alpha)}(x)$ and Its Convergence Analysis}

The main goal of this section is to introduce the following theorems to derive an approximate formula of the 
fractional derivatives of the generalized Laguerre polynomials and study the truncating error and its convergence analysis.

Theorem 1 [23]

Let $u(x)$ be approximated by the generalized Laguerre polynomials as (11) and also suppose $v>0$ then, its Caputo fractional derivative can be written in the following form

$$
D^{v}\left(u_{m}(x)\right)=\sum_{i=\lceil\rceil\rceil k=\vee v\rceil}^{m} \sum_{i}^{i} c_{i, k}^{(v)} x^{k-v},
$$

where $w_{i, k}^{(v)}$ is given by

$$
w_{i, k}^{(v)}=\frac{(-1)^{k}}{\Gamma(k+1-v)}\left(\begin{array}{c}
i+\alpha \\
i-k
\end{array}\right)
$$

\section{Theorem 2}

The Caputo fractional derivative of order $v$ for the generalized Laguerre polynomials can be expressed in terms of the generalized Laguerre polynomials themselves in the following form

$$
D^{v} L_{i}^{(\alpha)}(x) \cong \sum_{k=v v\rceil}^{i} \sum_{j=0}^{k-\lceil v\rceil} \Omega_{i j k} L_{j}^{(\alpha)}(x), \quad i=\lceil v\rceil,\lceil v\rceil+1, \cdots, m,
$$

where $\Omega_{i j k}=\sum_{r=0}^{j} \frac{(-1)^{r+k}(j) ! \Gamma(\alpha+i+1) \Gamma(k+\alpha-v+r+1)}{r !(j-r) !(i-k) ! \Gamma(k-v+1) \Gamma(\alpha+k+1) \Gamma(\alpha+r+1)}$.

Proof. See [24].

Theorem 3 [25].

The error in approximating $D^{v} u(x)$ by $D^{v} u_{m}(x)$ is bounded by

$$
\begin{gathered}
\left|E_{T}(m)\right| \leq \sum_{i=m+1}^{\infty} c_{i} \Pi_{v}(i, j) \frac{(\alpha+1)_{j}}{j !} \mathrm{e}^{x / 2}, \quad \alpha \geq 0, x \geq 0, j=0,1, \cdots, \\
\left|E_{T}(m)\right| \leq \sum_{i=m+1}^{\infty} c_{i} \Pi_{v}(i, j)\left(2-\frac{(\alpha+1)_{j}}{j !}\right) \mathrm{e}^{x / 2}, \quad-1<\alpha \leq 0, x \geq 0, j=0,1, \cdots,
\end{gathered}
$$

where, $\Pi_{v}(i, j)=\sum_{k=\ulcorner v\rceil}^{i} \sum_{j=0}^{k-\lceil v\rceil} \Omega_{i j k}$ and $\left|E_{T}(m)\right|=\left|D^{v} u(x)-D^{v} u_{m}(x)\right|$.

\section{Solution of the Fractional Wave Equation}

Consider the fractional wave equation of type given in Equation (1) in the interval $(0,1)$. In order to use Laguerre collocation method, we first approximate $u(x, t)$ as

$$
u_{m}(x, t)=\sum_{i=0}^{m} u_{i}(t) L_{i}^{(\alpha)}(x)
$$

From Equations (1), (17) and Theorem 1, we have

$$
\sum_{i=0}^{m} \frac{\mathrm{d}^{2} u_{i}(t)}{\mathrm{d} t^{2}} L_{i}^{(\alpha)}(x)=d(x, t) \sum_{i=\lceil\urcorner\rceil k=\lceil v\rceil}^{m} \sum_{i}^{i} u_{i}(t) w_{i, k}^{(v)} x^{k-v}+s(x, t),
$$

we now collocate Equation (18) at $(m+1-\lceil v\rceil)$ points $x_{p}, \quad p=0,1, \cdots, m-\lceil v\rceil$ as

$$
\sum_{i=0}^{m} \ddot{u}_{i}(t) L_{i}^{(\alpha)}\left(x_{p}\right)=d\left(x_{p}, t\right) \sum_{i=\lceil\urcorner\rceil k=\lceil v\rceil}^{m} \sum_{i}^{i} u_{i}(t) w_{i, k}^{(v)} x_{p}^{k-v}+s\left(x_{p}, t\right) .
$$

For suitable collocation points we use roots of shifted Laguerre polynomial $L_{m+1-\lceil v\rceil}^{(\alpha)}(x)$.

Also, by substituting Equations (17) and (11) in the boundary conditions (3) we can obtain $\lceil v\rceil$ equations as 
follows

$$
\sum_{i=0}^{m} \ell_{i} u_{i}(t)=0, \quad \sum_{i=0}^{m} \epsilon_{i} u_{i}(t)=0
$$

where $\ell_{i}=L_{i}^{(\alpha)}(0)=\left(\begin{array}{c}i+\alpha \\ i\end{array}\right), \epsilon_{i}=L_{i}^{(\alpha)}(1)$.

Equation (19), together with $\lceil v\rceil$ equations of the boundary conditions (20), give $(m+1)$ of ordinary differential equations which can be solved, for the unknowns $u_{i}, i=0,1, \cdots, m$, using the finite difference method, as described in the following section.

\section{Numerical Results}

In this section, we implement the proposed method to solve FWE (1) with $v=1.8$, of the form

$$
u_{t t}(x, t)=d(x, t) D^{1.8} u(x, t)+s(x, t), \quad 0<x<1, \quad t>0,
$$

where the coefficient and the source functions are $d(x, t)=\Gamma(1.2) x^{1.8}$ and $s(x, t)=x^{2}(4 x-1) \mathrm{e}^{-t}$, the initial and Dirichlet conditions are $u(x, 0)=x^{2}(1-x), u_{t}(x, 0)=x^{2}(x-1), u(0, t)=u(1, t)=0$. The exact solution to this problem is $u(x, t)=x^{2}(1-x) \mathrm{e}^{-t}$.

We apply the method with $m=3$, and approximate the solution as follows

$$
u_{3}(x, t)=\sum_{i=0}^{3} u_{i}(t) L_{i}^{(\alpha)}(x) .
$$

Using Equation (19) we have

$$
\sum_{i=0}^{3} \ddot{u}_{i}(t) L_{i}^{(\alpha)}\left(x_{p}\right)=d\left(x_{p}, t\right) \sum_{i=2}^{3} \sum_{k=2}^{i} u_{i}(t) w_{i, k}^{(1.8)} x_{p}^{k-1.8}+s\left(x_{p}, t\right), \quad p=0,1
$$

where $x_{p}$ are roots of Laguerre polynomial $L_{2}^{(\alpha)}(x)$, i.e. $x_{0}=0.427124$ and $x_{1}=3.07288$.

By using Equations (20) and (22) we obtain the following system of ODEs

$$
\begin{gathered}
\ddot{u}_{0}(t)+k_{1} \ddot{u}_{1}(t)+k_{2} \ddot{u}_{3}(t)=R_{1} u_{2}(t)+R_{2} u_{3}(t)+s_{0}(t), \\
\ddot{u}_{0}(t)+k_{11} \ddot{u}_{1}(t)+k_{22} \ddot{u}_{3}(t)=R_{11} u_{2}(t)+R_{22} u_{3}(t)+s_{1}(t), \\
\ell_{0} u_{0}(t)+\ell_{1} u_{1}(t)+\ell_{2} u_{2}(t)+\ell_{3} u_{3}(t)=0, \\
\epsilon_{0} u_{0}(t)+\epsilon_{1} u_{1}(t)+\epsilon_{2} u_{2}(t)+\epsilon_{3} u_{3}(t)=0,
\end{gathered}
$$

where $\ell_{i}$ and $\epsilon_{i}, i=0, \cdots, 3$ are defined in (20) and

$$
\begin{aligned}
& k_{1}=L_{1}^{(\alpha)}\left(x_{0}\right), k_{2}=L_{3}^{(\alpha)}\left(x_{0}\right), k_{11}=L_{1}^{(\alpha)}\left(x_{1}\right), k_{22}=L_{3}^{(\alpha)}\left(x_{1}\right), \\
& R_{1}=d\left(x_{0}, t\right) w_{2,2}^{(1.8)} x_{0}^{0.2}, R_{2}=d\left(x_{0}, t\right)\left[w_{3,2}^{(1.8)} x_{0}^{0.2}+w_{3,3}^{(1.8)} x_{0}^{1.2}\right], \\
& R_{11}=d\left(x_{1}, t\right) w_{2,2}^{(1.8)} x_{1}^{0.2}, R_{22}=d\left(x_{1}, t\right)\left[w_{3,2}^{(1.8)} x_{1}^{0.2}+w_{3,3}^{(1.8)} x_{1}^{1.2}\right] .
\end{aligned}
$$

Now, in order to use FDM to solve the system (23)-(26), we will use the notations $t_{i}=i \tau$ to be the integration time $0 \leq t_{i} \leq T, \tau=T / N$, for $i=0,1, \cdots, N$. Define $u_{i}^{n}=u_{i}\left(t_{n}\right), s_{i}^{n}=s_{i}\left(t_{n}\right)$. Then, the system (23)-(26), will discretize in time and take the following form

$$
\begin{gathered}
\frac{u_{0}^{n+1}-2 u_{0}^{n}+u_{0}^{n-1}}{\tau^{2}}+k_{1} \frac{u_{1}^{n+1}-2 u_{1}^{n}+u_{1}^{n-1}}{\tau^{2}}+k_{2} \frac{u_{3}^{n+1}-2 u_{3}^{n}+u_{3}^{n-1}}{\tau^{2}}=R_{1} u_{2}^{n+1}+R_{2} u_{3}^{n+1}+s_{0}^{n+1}, \\
\frac{u_{0}^{n+1}-2 u_{0}^{n}+u_{0}^{n-1}}{\tau^{2}}+k_{11} \frac{u_{1}^{n+1}-2 u_{1}^{n}+u_{1}^{n-1}}{\tau^{2}}+k_{22} \frac{u_{3}^{n+1}-2 u_{3}^{n}+u_{3}^{n-1}}{\tau^{2}}=R_{11} u_{2}^{n+1}+R_{22} u_{3}^{n+1}+s_{1}^{n+1}, \\
\ell_{0} u_{0}^{n+1}+\ell_{1} u_{1}^{n+1}+\ell_{2} u_{2}^{n+1}+\ell_{3} u_{3}^{n+1}=0,
\end{gathered}
$$




$$
\epsilon_{0} u_{0}^{n+1}+\epsilon_{1} u_{1}^{n+1}+\epsilon_{2} u_{2}^{n+1}+\epsilon_{3} u_{3}^{n+1}=0,
$$

we can write the above system (27)-(30) in the following matrix form as follows

$$
\begin{aligned}
& \left(\begin{array}{cccc}
1 & k_{1} & -\tau^{2} R_{1} & k_{2}-\tau^{2} R_{2} \\
1 & k_{11} & -\tau^{2} R_{11} & k_{22}-\tau^{2} R_{22} \\
\ell_{0} & \ell_{1} & \ell_{2} & \ell_{3} \\
\epsilon_{0} & \epsilon_{1} & \epsilon_{2} & \epsilon_{3}
\end{array}\right)\left(\begin{array}{l}
u_{0} \\
u_{1} \\
u_{2} \\
u_{3}
\end{array}\right)^{n+1} \\
& =\left(\begin{array}{cccc}
2 & 2 k_{1} & 0 & 2 k_{2} \\
2 & 2 k_{11} & 0 & 2 k_{22} \\
0 & 0 & 0 & 0 \\
0 & 0 & 0 & 0
\end{array}\right)\left(\begin{array}{l}
u_{0} \\
u_{1} \\
u_{2} \\
u_{3}
\end{array}\right)^{n}-\left(\begin{array}{cccc}
1 & k_{1} & 0 & k_{2} \\
1 & k_{11} & 0 & k_{22} \\
0 & 0 & 0 & 0 \\
0 & 0 & 0 & 0
\end{array}\right)\left(\begin{array}{l}
u_{0} \\
u_{1} \\
u_{2} \\
u_{3}
\end{array}\right)^{n-1}+\tau^{2}\left(\begin{array}{c}
s_{0} \\
s_{1} \\
0 \\
0
\end{array}\right)^{n+1} .
\end{aligned}
$$

The above system can be rewritten in a matrix form as follows

$$
A U^{n+1}=B U^{n}-C U^{n-1}+S^{n+1} \text {, or } U^{n+1}=A^{-1} B U^{n}-A^{-1} C U^{n-1}+A^{-1} S^{n+1},
$$

where $U^{n}=\left(u_{0}^{n}, u_{1}^{n}, u_{2}^{n}, u_{3}^{n}\right)^{\mathrm{T}}$ and $S^{n}=\left(\tau^{2} s_{0}^{n}, \tau^{2} s_{1}^{n}, 0,0\right)^{\mathrm{T}}$.

The obtained numerical results by means of the proposed method are shown in Table 1 and (Figure 1 and Figure 2). In Table 1, the absolute errors between the exact solution $u_{e x}$ and approximate solution $u_{\text {approx }}$ at $m=$ 3 and $m=5$ with the final time $T=2$ are given. Also, in Figure 1 and Figure 2, comparison between the exact solution and the approximate solution at $T=1$ with time step $\tau=0.0025$, and $m=3, m=5$ respectively are presented.

\section{Conclusion and Remarks}

This article is devoted to introducing an accurate numerical technique for solving the fractional wave equation. The prosed method depends on the approximate formula for the Caputo fractional derivative of the generalized Laguerre polynomials derived above. In the proposed method, the properties of the Laguerre polynomials are used to reduce FWE to solve a system of ODEs which solved by using FDM. The results show that the introduced algorithm converges as the number of $m$ terms is increased. The solution is expressed as a truncated Laguerre series and so it can be easily evaluated for arbitrary values of time using any computer program without any computational effort. Although we only considered a model problem in this paper, the main idea and the

Table 1. The absolute error between the exact solution and
the approximate solution at $m=3, m=5$ and $T=2$.
\begin{tabular}{ccc}
\hline$x$ & $\left|u_{e x}-u_{\text {opprox }}\right|$ at $m=3$ & $\left|u_{e x}-u_{\text {opprox }}\right|$ at $m=5$ \\
\hline 0.0 & $3.753964 \mathrm{e}-03$ & $3.147852 \mathrm{e}-05$ \\
0.1 & $4.756213 \mathrm{e}-03$ & $5.587900 \mathrm{e}-05$ \\
0.2 & $5.159753 \mathrm{e}-03$ & $4.524873 \mathrm{e}-05$ \\
0.3 & $1.987123 \mathrm{e}-03$ & $8.258963 \mathrm{e}-05$ \\
0.4 & $7.032516 \mathrm{e}-03$ & $7.654872 \mathrm{e}-05$ \\
0.5 & $3.147852 \mathrm{e}-03$ & $6.000214 \mathrm{e}-05$ \\
0.6 & $2.954621 \mathrm{e}-03$ & $6.753951 \mathrm{e}-05$ \\
0.7 & $0.753951 \mathrm{e}^{-03}$ & $5.987456 \mathrm{e}-05$ \\
0.8 & $1.654789 \mathrm{e}-03$ & $2.225544 \mathrm{e}-05$ \\
0.9 & $5.123456 \mathrm{e}-03$ & $0.159753 \mathrm{e}-05$ \\
1.0 & $6.002547 \mathrm{e}-03$ & $0.025467 \mathrm{e}-05$
\end{tabular}






Figure 1. Comparison between the exact solution and the approximate solution at $T=1$ with $\tau=0.0025, m=3$.

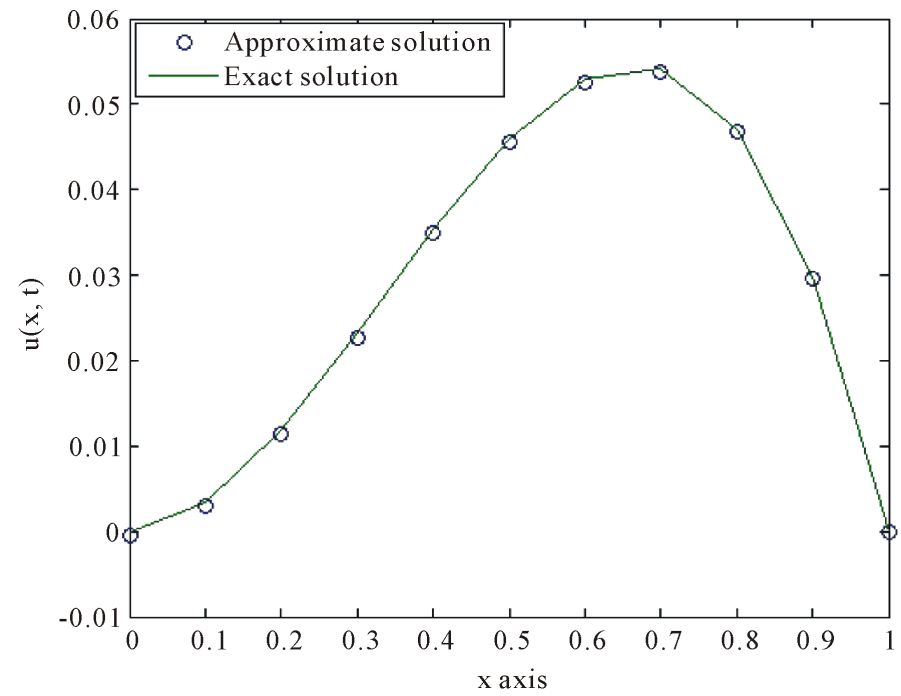

Figure 2. Comparison between the exact solution and the approximate solution at $T=1$ with $\tau=0.0025, m=5$.

used techniques are also applicable to many other problems. It is evident that the overall errors can be made smaller by adding new terms from the series (21). In the end, from our numerical results using the proposed method, we can see that, the solutions are in excellent agreement with the exact solution. All computations are made by Matlab.

\section{References}

[1] Miller, K.S. and Ross, B. (1993) An Introduction to the Fractional Calculus and Fractional Differential Equations. John Wiley and Sons, New York.

[2] Podlubny, I. (1999) Fractional Differential Equations. Academic Press, New York.

[3] Sweilam, N.H., Khader, M.M. and Al-Bar, R.F. (2007) Numerical Studies for a Multi-Order Fractional Differential Equation. Physics Letters A, 371, 26-33. http://dx.doi.org/10.1016/j.physleta.2007.06.016

[4] Hashim, I., Abdulaziz, O. and Momani, S. (2009) Homotopy Analysis Method for Fractional IVPs. Communications in Nonlinear Science and Numerical Simulations, 14, 674-684. http://dx.doi.org/10.1016/j.cnsns.2007.09.014 
[5] Funaro, D. (1992) Polynomial Approximation of Differential Equations, Springer Verlag, New York.

[6] Khader, M.M. (2011) On the Numerical Solutions for the Fractional Diffusion Equation. Communications in Nonlinear Science and Numerical Simulations, 16, 2535-2542. http://dx.doi.org/10.1016/j.cnsns.2010.09.007

[7] Sweilam, N.H., Khader, M.M. and Adel, M. (2014) Chebyshev Pseudo-Spectral Method for Solving Fractional Advection-Dispersion Equation. Applied Mathematics, 5, 3240-3248. http://dx.doi.org/10.4236/am.2014.519301

[8] Sweilam, N.H., Khader, M.M. and Mahdy, A.M.S. (2012) Numerical Studies for Fractional-Order Logistic Differential Equation with Two Different Delays. Journal of Applied Mathematics, 2012, Article ID: 764894, 14 p.

[9] Sweilam, N.H. and Khader, M.M. (2010) A Chebyshev Pseudo-Spectral Method for Solving Fractional Integro-Differential Equations. ANZIAM, 51, 464-475. http://dx.doi.org/10.1017/S1446181110000830

[10] Sweilam, N.H., Khader, M.M. and Adel, M. (2014) Numerical Simulation of Fractional Cable Equation of Spiny Neuronal Dendrites. Journal of Advanced Research (JAR), 5, 253-259. http://dx.doi.org/10.1016/j.jare.2013.03.006

[11] Smith, G.D. (1965) Numerical Solution of Partial Differential Equations. Oxford University Press, New York.

[12] Jafari, H. and Daftardar-Gejji, V. (2006) Solving Linear and Nonlinear Fractional Diffusion and Wave Equations by Adomian Decomposition Method. Applied Mathematics and Computation, 180, 488-497. http://dx.doi.org/10.1016/j.amc.2005.12.031

[13] Sweilam, N.H., Khader, M.M. and Nagy, A.M. (2011) Numerical Solution of Two-Sided Space-Fractional Wave Equation Using Finite Difference Method. Journal of Computational and Applied Mathematics, 235, 2832-2841. http://dx.doi.org/10.1016/j.cam.2010.12.002

[14] Sweilam, N.H., Khader, M.M. and Adel, M. (2012) On the Stability Analysis of Weighted Average Finite Difference Methods for Fractional Wave Equation. Fractional Differential Calculus, 2, 17-29. http://dx.doi.org/10.7153/fdc-02-02

[15] Chen, S., Liu, F., Zhuang, P. and Anh, V. (2009) Finite Difference Approximations for the Fractional Fokker-Planck Equation. Applied Mathematical Modelling, 33, 256-273. http://dx.doi.org/10.1016/j.apm.2007.11.005

[16] Khader, M.M. (2013) Numerical Treatment for Solving the Perturbed Fractional PDEs Using Hybrid Techniques. Journal of Computational Physics, 250, 565-573. http://dx.doi.org/10.1016/j.jcp.2013.05.032

[17] Lubich, Ch. (1986) Discretized Fractional Calculus. SIAM Journal on Mathematical Analysis, 17, 704-719. http://dx.doi.org/10.1137/0517050

[18] Meerschaert, M.M. and Tadjeran, C. (2006) Finite Difference Approximations for Two-Sided Space-Fractional Partial Differential Equations. Applied Numerical Mathematics, 56, 80-90. http://dx.doi.org/10.1016/j.apnum.2005.02.008

[19] Liu, F., Zhuang, P. and Burrage, K. (2012) Numerical Methods and Analysis for a Class of Fractional Advection-Dispersion Models. Computer and Mathematics with Application, 64, 2990-3007. http://dx.doi.org/10.1016/j.camwa.2012.01.020

[20] Canuto, C., Hussaini, M.Y., Quarteroni, A. and Zang, T.A. (2006) Spectral Methods. Springer-Verlag, New York.

[21] Xu, C.-L. and Guo, B.-Y. (2002) Laguerre Pseudo-Spectral Method for Non-Linear Partial Differential Equations. Journal of Computational Mathematics, 20, 413-428.

[22] Wang, L. and Guo, B.Y. (2006) Stair Laguerre Pseudo-Spectral Method for Differential Equations on the Half Line. Advances in Computational Mathematics, 25, 305-322. http://dx.doi.org/10.1007/s10444-003-7608-6

[23] Khader, M.M. (2013) The Use of Generalized Laguerre Polynomials in Spectral Methods for Fractional-Order Delay Differential Equations. Journal of Computational and Nonlinear Dynamics, 8, Article ID: 041018.

[24] Doha, E.H., Bhrawy, A.H. and Ezz-Eldien, S.S. (2011) Efficient Chebyshev Spectral Methods for Solving Multi-Term Fractional Orders Differential Equations. Applied Mathematical Modelling, 35, 5662-5672. http://dx.doi.org/10.1016/j.apm.2011.05.011

[25] Khader, M.M. and Babatin, M.M. (2013) On Approximate Solutions for Fractional Logistic Differential Equation. Mathematical Problems in Engineering, 2013, Article ID: 391901. http://dx.doi.org/10.1155/2013/391901 